\title{
Influence of a Magnetic Guide Field on Self-Injection in Wakefield Acceleration
}

\author{
Alain Bourdier, Guillaume Girard, Sébastien Rassou, Xavier Davoine, Mathieu Drouin \\ CEA, DAM, DIF, Arpajon, France \\ Email: alain.bourdier@gmail.com
}

Received September 28, 2012; revised October 28, 2012; accepted November 7, 2012

\begin{abstract}
The influence of an external static field applied in the direction of propagation of a high intensity driving laser pulse on the electron trapping in laser wakefield acceleration is explored. It is shown that, in the case of self-injection, the electric charge accelerated can be enhanced in some physical situations.
\end{abstract}

Keywords: Laser Wakefield Acceleration; Magnetic Guide Field; Electron Injection; Self-Trapping

\section{Introduction}

Particle accelerators have important applications in many fields, from medicine to high energy physics [1-6]. Laser-plasma accelerator have a decisive advantage over conventional accelerators: plasma supports electric fields orders of magnitude higher than the breakdown-limited field in radio-frequency cavities of conventional linacs. The acceleration gradients in conventional accelerators are limited to a few tens of $\mathrm{MV} / \mathrm{m}$, while they can reach and even exceed $100 \mathrm{GV} / \mathrm{m}$ in plasma. Ultra short light pulses that can be generated with a plasma accelerator are powerful tools for time resolved studies of molecular and atomic dynamics [7].

Among the various laser-plasma accelerator concepts, the laser-wakefield accelerator (LWFA) $[1,8]$ is the most promising [2]. One of the key applications of laserplasma interaction is particle acceleration based on the excitation of a strong plasma wakefield by a laser. As low density plasma is always considered, the phase velocity of the plasma wake is close to the speed of light, so the charged particles which are loaded in the wake can be accelerated to very high energy.

One of the most critical issues in the LWFA process may be how to control and enhance the beam energy and the charge. It is shown in this article that a static longitudinal magnetic field can enhance particle trapping.

When a laser pulse propagates in plasma, the ponderomotive force which is proportional to the intensity gradient of the pulse pushes electrons forwards and sideways. Because the ions are much heavier than the electrons and so do not respond to the ponderomotive force, the electrons are dragged back towards their original position by the space charge field and start to oscillate, cre- ating a plasma wave and the so-called wakefield. When the intensity of the pulse is high enough the "bubble" regime is reached $[9,10]$. Hence, a cavity structure generally called bubble is driven in low density plasma by the laser pulse through the ponderomotive force. The laser pulse can excite a plasma wave in different ways. The excitation is most effective when the laser pulse is shorter than the plasma wavelength $\lambda_{p}=2 \pi c / \omega_{p}$ where $\omega_{p}$ is the plasma frequency. Usually, electrons oscillating in the plasma wave under the wave-breaking limit cannot be accelerated by the wakefield since they are out of phase with it. If the laser intensity reaches a threshold value, some electrons can oscillate so fast in the plasma wave that they can reach the wave velocity. In this case, these electrons overtake the wave and wave breaking occurs. Then, they stop to oscillate in the wave and are injected in the wakefield and start to propagate with it. As the electron reaches the highest velocity at the back of the bubble, they are injected at this position in the bubble. Wave breaking turns out to be important as it leads to abundant self-trapping of electrons in the wakefield. However, the electron beams created this way do not usually have the stability and reproducibility that are required for applications [11]. This is because the mechanism responsible for injecting electrons into the wakefield is based on highly nonlinear phenomena, and is therefore hard to control. It is well known that trapping of the background electrons begins much below the longitudinal wave-breaking limit [12-14]. The transverse wave-breaking regime which has to be studied with a two dimensional approach is the situation where a static magnetic field should play an important role. It was already shown by Hur, Gupta, and Suk [14] and by Vieira 
et al. [15] in the case of a transverse constant magnetic field that a static magnetic field can play an important part in order to control the electron injection in the accelerating cavity, it should play a role similar to the one of a perturbing counter propagating wave. As a consequence, we started a deeper exploration of the positive effect of a static magnetic field which has been chosen, in this work, to be applied in parallel with the propagation direction of the driving laser pulse. Following a paper previously published by Hur, Gupta, and Suk [14], the effect of such a field on the trapped beam is studied again in this article. The idea was to verify if the magnetic field can be considered as an important controlling knob for the trapped charge in the case of self-injection.

\section{Electron Injection Enhanced by a Static Magnetic Field-PIC Code Simulation Results}

The influence of a constant homogeneous magnetic guide field on LWFA is studied here with a code. Numerical simulations were conducted using the two-dimensional PIC code CALDER [16]. Their results were compared to those obtained by Hur, Gupta, and Suk with code XOOPIC [17]. In their paper Hur, Gupta, and Suk claim that a static magnetic field applied in parallel with the direction of propagation of the driving laser pulse enhances the particle trapping in the first bubble. They show from two-dimensional PIC code simulations that the total charge of the trapped beam and its maximum energy increase with the magnitude of the guide field.

In order to test our code, the simulation domain considered in this work was chosen to be as close as possible to the one considered by Hur, Gupta, and Suk. The wavelength of the laser was assumed to be $\lambda=1 \mu \mathrm{m}$ in all the simulations. A moving window was employed to reduce the computational time. It defines the simulation domain which was divided in nearly the same number of cells as in the simulations performed by Hur, Gupta, and Suk. The simulation box considered here, which drifts with the moving window, is $80 \mu \mathrm{m}$ in the laser propagation direction $(z)$ and $120 \mu \mathrm{m}$ in the transverse direction $(x)$. A trapezoidal electronic density profile in the longitudinal direction is assumed, with one homogeneous slab surrounded by two density gradients, $L_{g}$ is the density gradient length.

In order to compare our results to their results, the same parameters were considered: the plasma density was assumed to be: $n=3.4 \times 10^{18} \mathrm{~cm}^{-3}$, and the normalized vector potential of the laser pulse: $a=e A / m c^{2}=3.5$. The pulse duration was $\Delta t=38 \mathrm{fs}$ and the spot size of the laser was assumed to be $12 \mu \mathrm{m}$ large. When using CALDER, the 2D1/2 version was used.

As some parameters of the simulations performed by
Hur, Gupta, and Suk are not specified in their paper, different situations were considered. No significant charge trapped in the first bubble was found considering the physical parameters chosen by them. Electrons are found to be mainly trapped in the second and third bubble (Figure 1). The simulation results shown just below correspond to a time which is normalized to the laser frequency $\hat{t}=t / \omega_{0}$. Normalized space variables:

$$
\hat{i}=\left(\omega_{0} / c\right) i \quad(i=x, z)
$$

and a normalized momentum: $\hat{p}_{i}=p_{i} / m c$ are also introduced.

This result is confirmed when calculating the electron energy distribution in the three first bubbles (Figure 2).

In this article, the number of particles, $N$, expressed in $10^{6}$ particles per $\mathrm{MeV}$ is calculated for a $8 \mu \mathrm{m}$ thick plasma in the direction $(y)$. This assumption had to be done as 2D simulations have been performed.

The influence of the plasma density gradient $L_{g}$ was

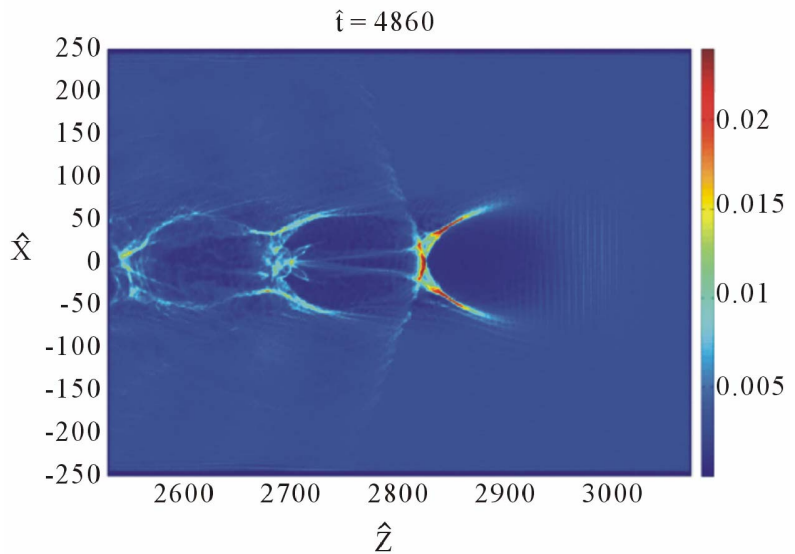

Figure 1. Electron density as a function of the longitudinal position from 2D PIC simulations. $a=3.5, n=3.4 \times 10^{18} \mathrm{~cm}^{-3}$.

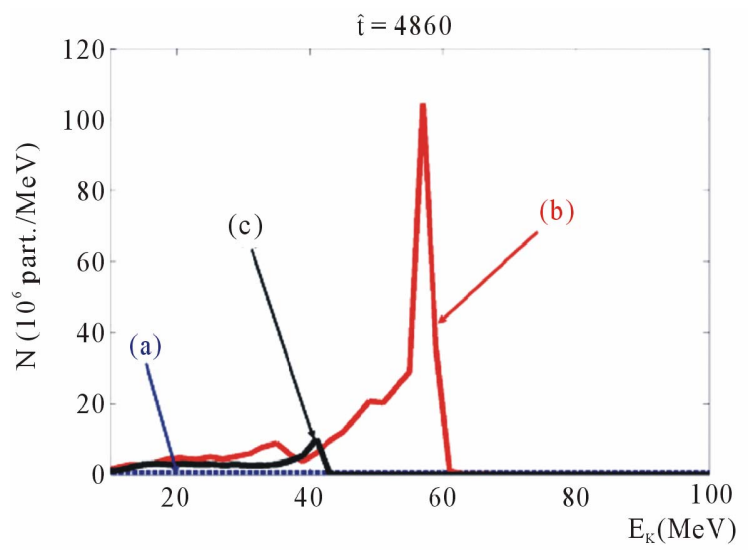

Figure 2. Electron energy distribution in the three first bubbles. $a=3.5, n=3.4 \times 10^{18} \mathrm{~cm}^{-3}, L_{g}=200 \mu \mathrm{m}$. (a) First bubble; (b) Second bubble; (c) Third bubble. 
explored first. Simulations show that the plasma density gradient has a strong influence on the electron energy distribution in the second bubble. Still no one of the different values of $L_{g}$ considered allows particles to be trapped in the first bubble.

The influence of the magnetic field on the distribution in the second bubble was also explored. It has been shown that the initial constant homogeneous guide magnetic field, when inferior to $250 \mathrm{~T}$, has almost no influence on the electron energy distribution in the second bubble.

The time step used in our simulations is just below the limit given by the Courant condition

$$
\Delta t \leq \sqrt{1 /\left[1 /(\Delta x)^{2}+1 /(\Delta y)^{2}\right]} .
$$

This upper limit is in our case:

$$
\Delta t_{L}=0.310 \omega_{0}^{-1}(\approx 0.16 \mathrm{fs}),
$$

and the time step used in our simulations is

$$
\Delta t=0.25 \omega_{0}^{-1}(\approx 0.13 \mathrm{fs})
$$

while the size of meshes is given by:

$$
\Delta z=0.313 c / \omega_{0}\left(\approx 5 \times 10^{-2} \mu \mathrm{m}\right)
$$

and

$$
\Delta x=2.5 c / \omega_{0}(\approx 0.4 \mu \mathrm{m}) .
$$

In order to check if the results published by Hur, Gupta, and Suk, who observed electrons trapped in the first bubble, were due to a bad optimization of their time step, simulations with smaller time steps were performed as a slower group velocity might have led to some trapping in the first bubble. No electron trapped in the first bubble was observed even when using a time step ten times smaller than the one usually used in the PIC simulations, that is to say the one chosen just below the Courant condition.

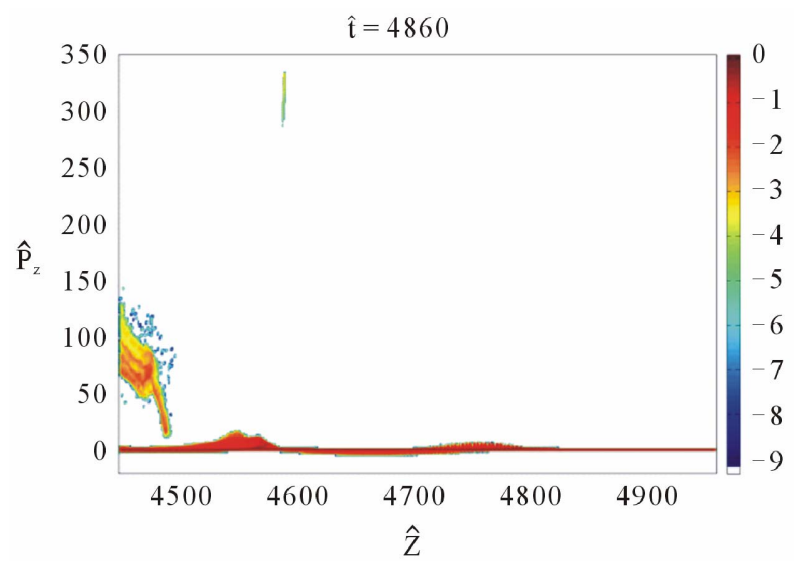

Figure 3. Electron longitudinal momentum distribution as a function of the longitudinal position. $a=7, n=3.4 \times 10^{18} \mathrm{~cm}^{-3}$, $B_{0}=0$.

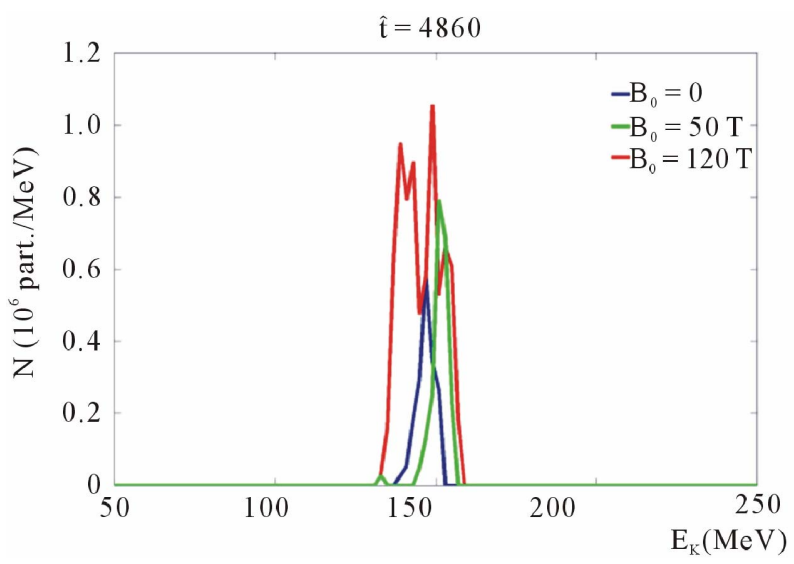

Figure 4. Electron energy distribution in the first bubble. $a=$ $7, n=3.4 \times 10^{18} \mathrm{~cm}^{-3}$.

For this value of the intensity of the laser pulse $(a=3.5)$ higher electron densities up to $1 \times 10^{19} \mathrm{~cm}^{-3}$ were considered in order to diminish the group velocity of the wave. No electron trapping in the first bubble was observed.

Then, the laser intensity was increased in order to try to have some self injection in the first bubble. Figures 3 and 4 show that some electrons are trapped in the first bubble when $a=7$.

Figure 4 shows that a very strong magnetic field along the direction of propagation of the wave allows the wakefield to trap more electrons in the first bubble.

When $B_{0}=0$ the charge trapped in the bubble is close to $\delta q=0.56$ picocoulombs, when $B_{0}=50 \mathrm{~T}$ the charge is $\delta q$ $=0.86$ picocoulombs and when $B_{0}=120 \mathrm{~T}$ one has $\delta q=$ 2.4 picocoulombs. Between $B_{0}=0$ and $B_{0}=120 \mathrm{~T}$ the trapped charge is multiplied by $\sim 4.3$. In this case, the magnetic field has no visible effect on the dimension of the bubble. Although these charges are very weak they show a trend: the charge increases with the magnitude of the static magnetic field. The non-relativistic plasma frequency in the presence of a magnetic field can be approximated by $\omega_{m} \approx\left(\omega_{p}^{2}+\Omega^{2}\right)^{1 / 2}$ where $\omega_{m}$ and $\omega_{p}$ represent the plasma frequencies of the magnetized plasma and the unmagnetized plasma, respectively. The cyclotron frequency is defined by $\Omega=e B_{0} / m$. When $B_{0}=120 \mathrm{~T}$, one has $(1 / 2) \Omega^{2} / \omega_{p}^{2} \approx 2 \times 10^{-2}$ which is too small to give any significant modification in the plasma frequency, as a consequence, the plasma itself behaves as if there were no magnetic field.

Figure 5 displays the magnetic field density along the direction of propagation of the wave for an initial constant magnetic field $B_{0}=120 \mathrm{~T}$. It shows that some magnetic field along the $z$-axis is built up close to the back of the cavity.

No magnetic field is created when the initial static magnetic field is zero.

Figure 6 shows the distributions in momentum along 


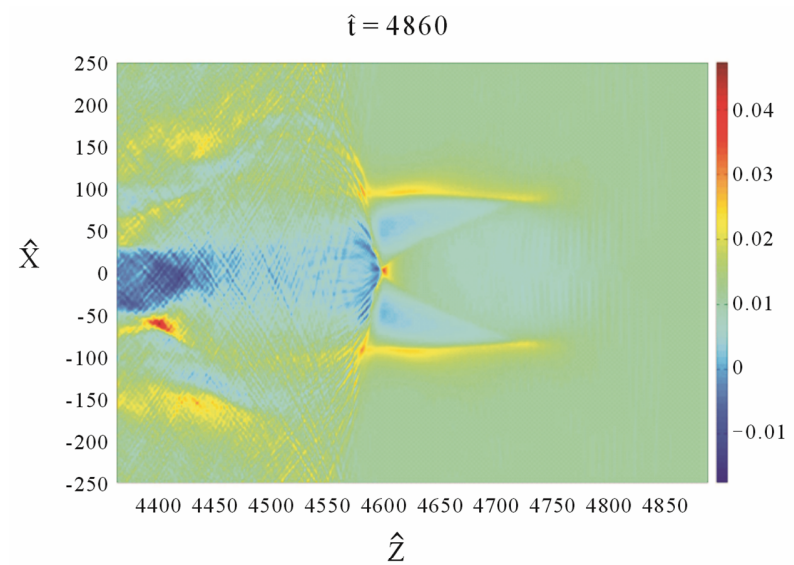

Figure 5. Map of the longitudinal magnetic field. $a=7$, $n=3.4 \times 10^{18} \mathrm{~cm}^{-3}, B_{0}=120 \mathrm{~T}$.

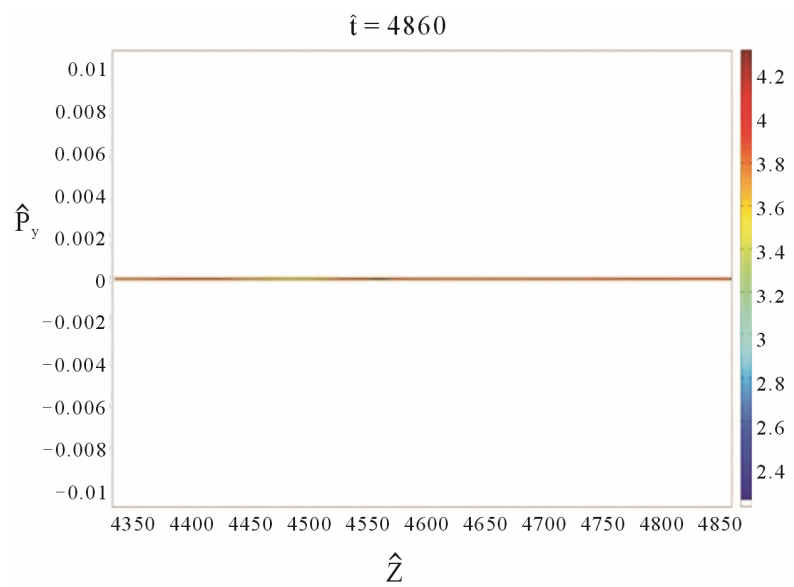

(a)

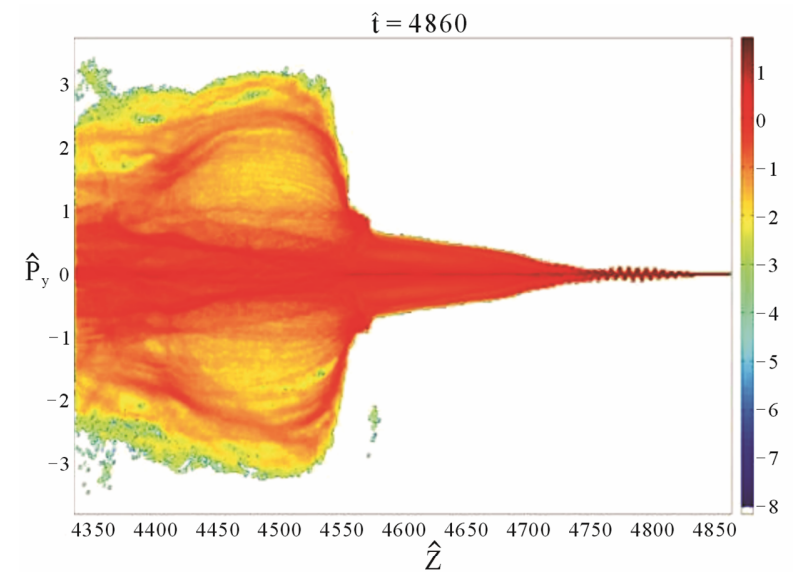

(b)

Figure 6. Electron momentum along the $y$ axis distribution in the first bubble. $a=7, n=3.4 \times 10^{18} \mathrm{~cm}^{-3}$. (a) $B_{0}=0$; (b) $B_{0}$ $=120 \mathrm{~T}$.

the $y$ axis versus $z$ when $B_{0}=0$ and $B_{0}=120 \mathrm{~T}$.

Due to the static magnetic field, electrons which drift on the rim of the cavity turn around the back of the bubble.
A transverse current is built up (Figure 6(b)). Consequently, a strong static magnetic field is created along the $z$ axis. Thus, a relatively small amplitude initial magnetic field is enough to build up a strong one. When $a=7$ and $a$ $=7.5$, Figures 4 and 7 show that the total charge of the trapped electrons in the first bubble increases with the initial magnitude of the magnetic field. This result is quite similar to the one obtained by Hur, Gupta, and Suk [14].

In this case $(a=7.5)$, when $B_{0}=0$ the trapped charge in the high energy peak is $\delta q=2.85$ picocoulombs, when $B_{0}$ $=120 \mathrm{~T}$, one has $\delta q_{B}=7.7$ picocoulombs, the significant parameter is $r=\delta q_{B} / \delta q \approx 2.7$. When this strong static magnetic field is applied the accelerated charge is multiplied by almost three.

Then, a higher wave intensity was considered: $a=8$. Figure 8(a) shows that a high energy peak close to 250 $\mathrm{MeV}$ is created. Figures 8(b) and (c) show that the static magnetic field has no significant influence on the bubble size. The correction to the plasma frequency due to the initial magnetic field is still $(1 / 2) \Omega^{2} / \omega_{p}^{2} \approx 2 \times 10^{-2}$. At $\hat{t}=4860$, when the initial static magnetic field has a magnitude of $120 \mathrm{~T}$, the longitudinal component of the magnetic field reaches about $350 \mathrm{~T}$ (Figure 9), then $(1 / 2) \Omega^{2} / \omega_{p}^{2} \approx 0.35$. This ratio is made smaller when taking into account the relativistic mass increase of the electrons. Thus, no significant deformation of the cavity due to the magnetic field is expected and no deformation is observed.

In this case, many more electrons turn around the magnetized cavity, then a more intense transverse current is produced.

Figure 9 shows the evolution of the very strong $z$ component of the magnetic field built up by the electrons turning around the neck of the bubble versus time for two initial values of $B_{0}$.

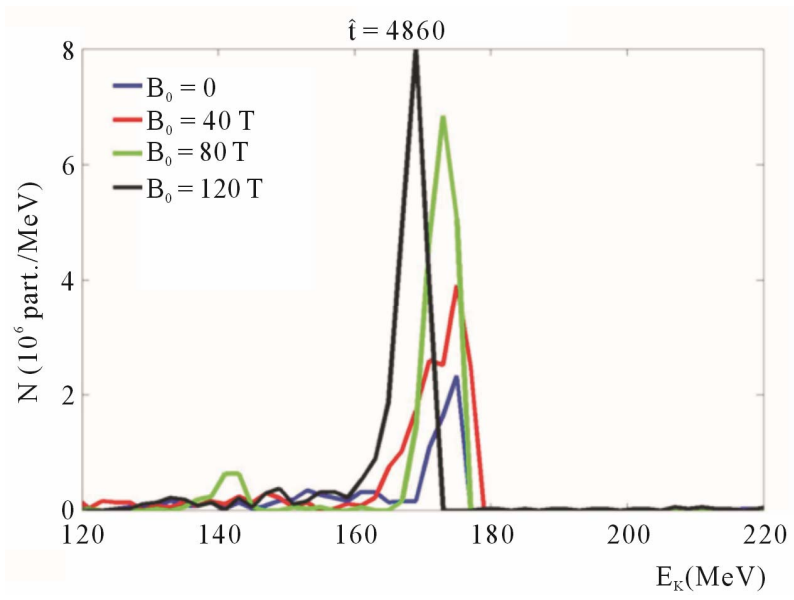

Figure 7. Electron energy distribution in the first bubble in a neighborhood of the high energy peak. for different values of the initial static magnetic field $a=7.5, n=3.4 \times 10^{18} \mathrm{~cm}^{-3}$. 


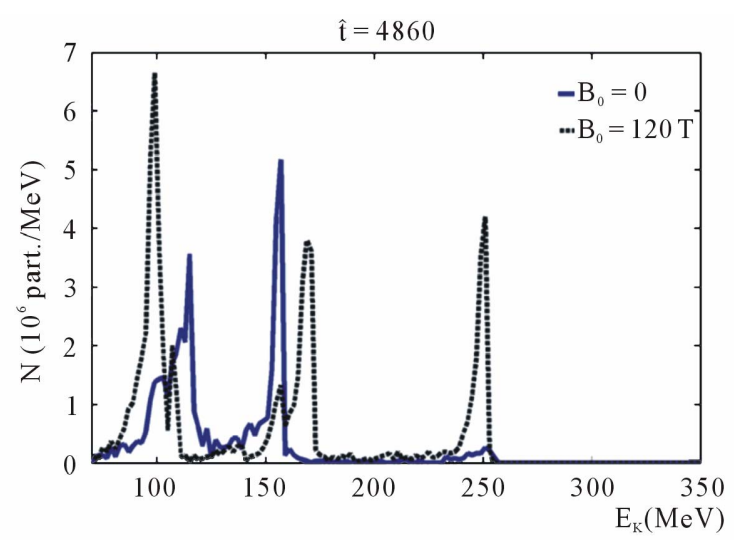

(a)

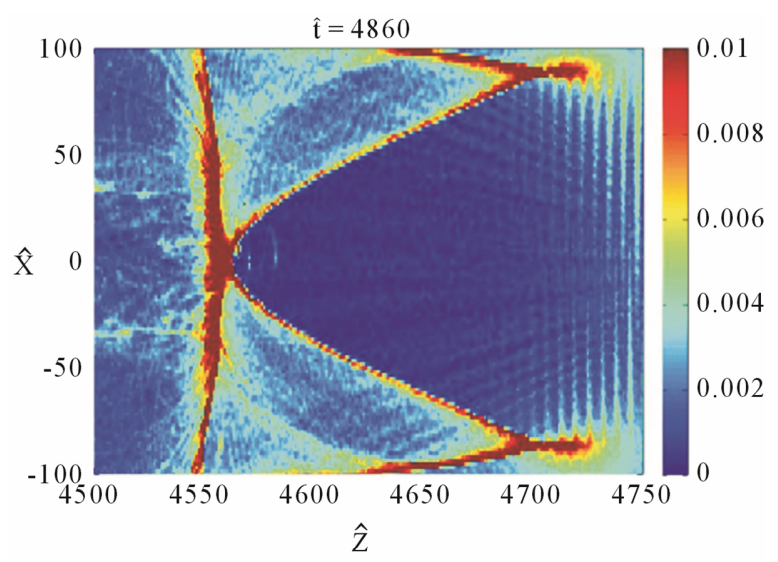

(b)

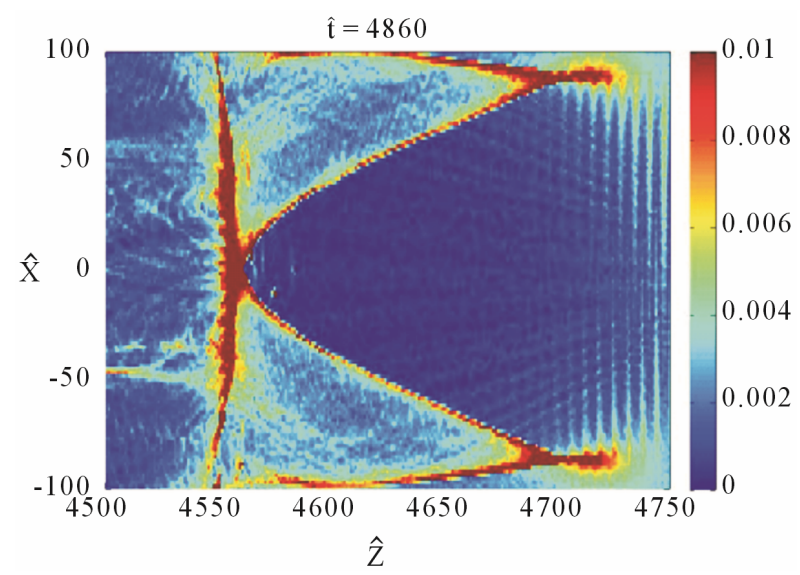

(c)

Figure 8. (a) Electron energy distribution in the first bubble; (b) Electron density when $B_{0}=0$; (c) Electron density when $B_{0}=120$ T. $a=8, n=3.4 \times 10^{18} \mathrm{~cm}^{-3}$.

A transverse magnetic field is also created. Figure 10 shows the $B_{x}$ component of the magnetic field close to the rear of the bubble.

Figure 11 shows the electron energy distribution for the trapped bunch close to the high energy peak displayed in Figure 8(a) for different values of $B_{0}$.

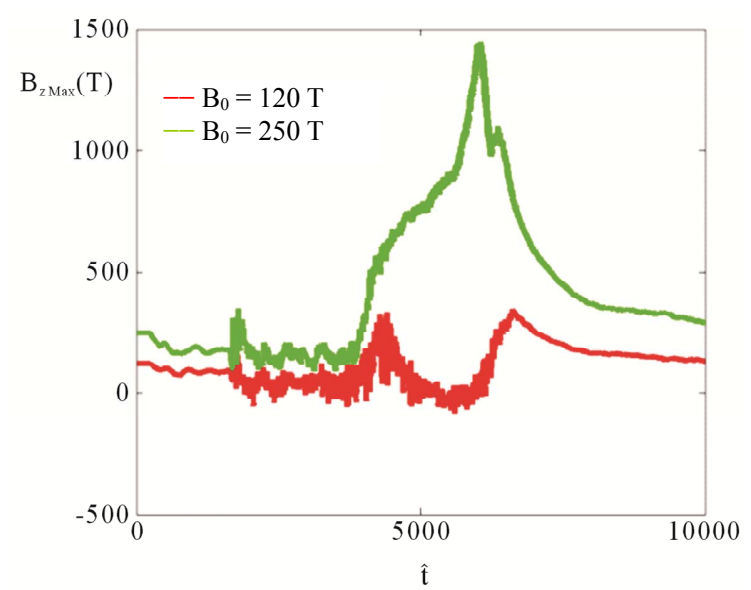

Figure 9. Longitudinal component of the magnetic field close to the rear of the bubble versus time. $a=8, n=3.4 \times$ $10^{18} \mathrm{~cm}^{-3}$.

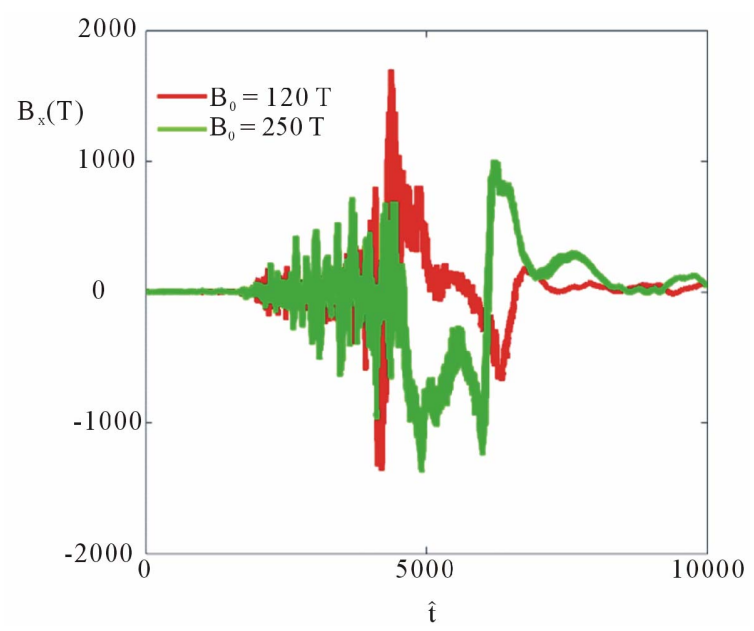

Figure 10. Evolution of the $x$ component of the magnetic field close to the rear of the bubble $a=8, n=3.4 \times 10^{18} \mathrm{~cm}^{-3}$.

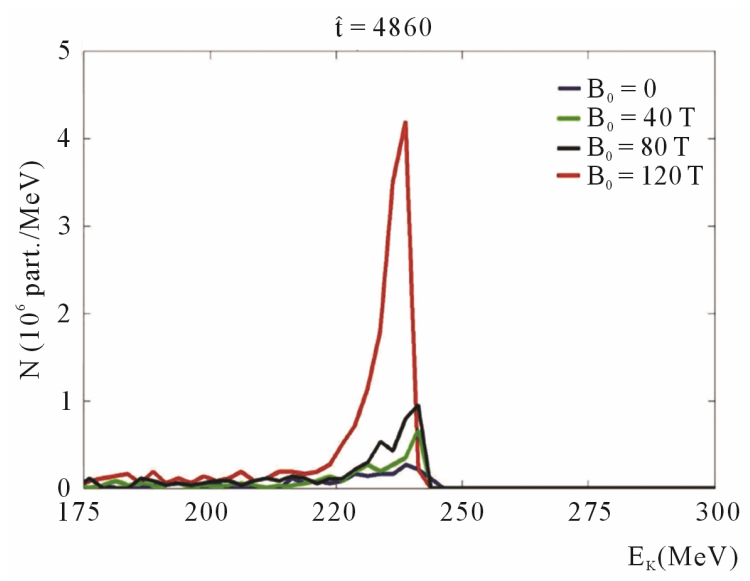

Figure 11. Electron energy distribution in the first bubble, for different values of the initial static magnetic field in a neighborhood of the high energy peak. $a=8, n=3.4 \times$ $10^{18} \mathrm{~cm}^{-3}$. 
When $B_{0}=0$, one has $\delta q=0.5$ picocoulombs and when $B_{0}=120 \mathrm{~T}$ one has $\delta q=4.6$ picocoulombs, the trapped charge is multiplied by $r=\delta q_{B} / \delta q \approx 9.2$.

The evolution of the electron energy distribution is similar to the one shown in Figure 3 in the article published by Hur, Gupta, and Suk. Many trajectories are bent by the magnetic field, keeping particles closer to the rear of the bubble. Then, the electron trapping is more likely to occur [18].

In conclusion, we have found again, for $a=7$ and $a=8$, results similar to those previously published by Hur, Gupta, and Suk for their electron density but for different values of the laser intensity.

More high energy particles are trapped in the high energy peak in the first bubble due to a strong magnetic field and to values of the pulse normalized vector potential close to $a=9$ (Figure 12).

When the intensity reaches $a=10$, many electrons are trapped in the first bubble even when there is no magnetic field (Figure 13).

The electron energy distribution is not very much affected by the magnetic field (Figure 14).

The number of trapped particles is close enough to the beam loading limit $[19,20]$, as a consequence the accelerated beam has a large energy spread with or without a strong magnetic field [21].

In this case, a strong longitudinal magnetic field is still built up.

For this very high intensity, electron trapping is no longer enhanced by a static longitudinal magnetic field and the static magnetic stops having a positive effect on the quality of the beam trapped in the first bubble.

\section{Conclusions}

The goal of this work was mainly to verify the very in-

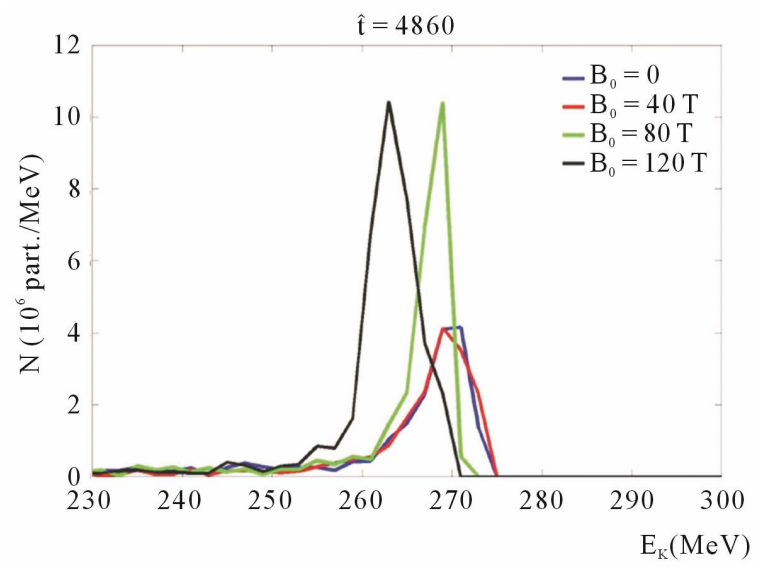

Figure 12. Electron energy distribution in the first bubble, for different values of the initial static magnetic field in a neighborhood of the high energy peak. $a=9, n=3.4 \times$ $10^{18} \mathrm{~cm}^{-3}$.

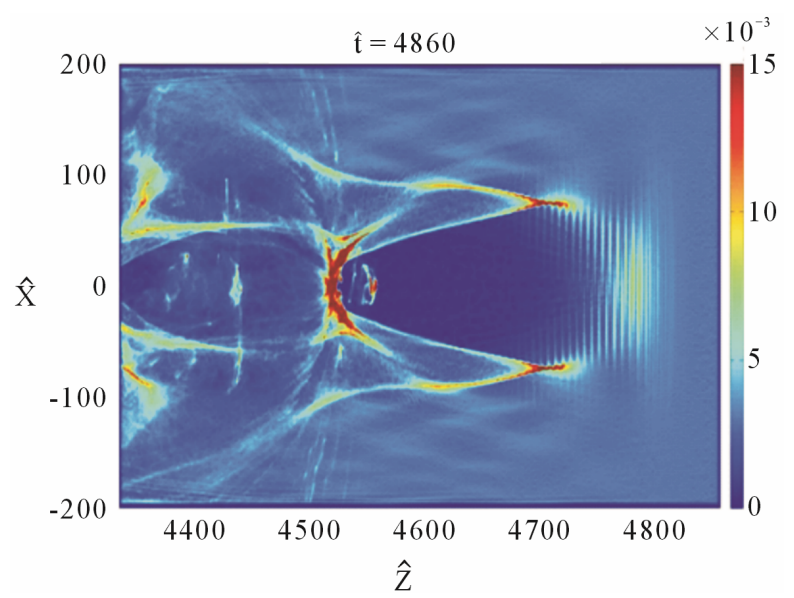

(a)

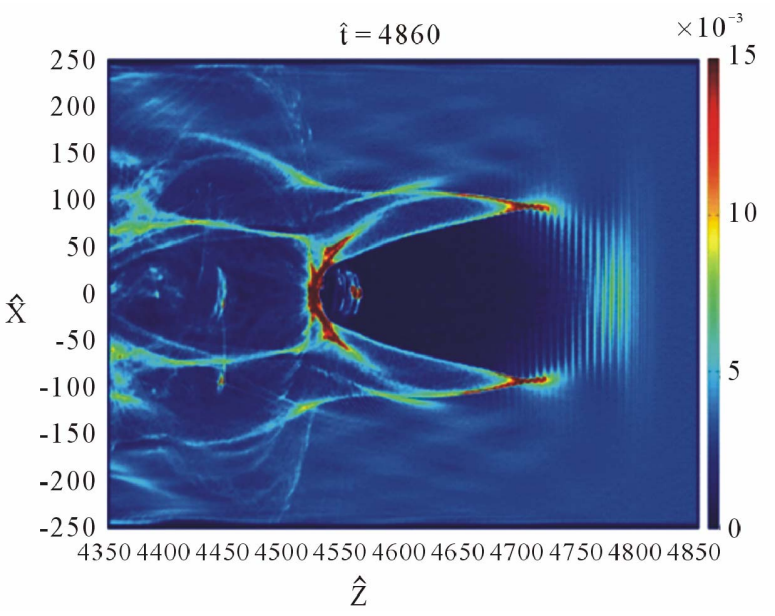

(b)

Figure 13. Electron density. $a=10, n=3.4 \times 10^{18} \mathrm{~cm}^{-3}$. (a) When $B_{0}=0$; (b) When $B_{0}=120 \mathrm{~T}$.

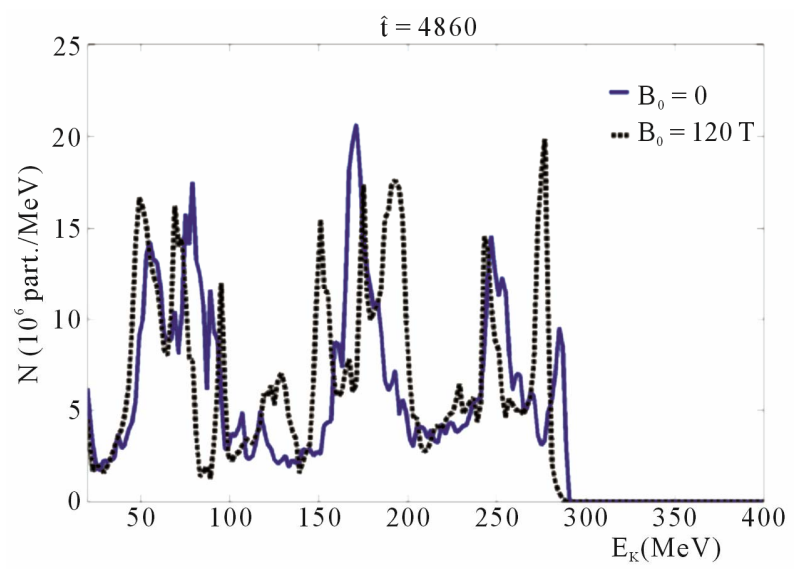

Figure 14. Electron energy distribution in the first bubble. $a$ $=10, \mathrm{n}=3.4 \times 10^{18} \mathrm{~cm}^{-3}$.

teresting results previously published by Hur, Gupta, and Suk [14] concerning the influence of a constant magnetic field in the enhancement of the charge trapped by the 
first bubble in the case of auto-injection. Although their results are not confirmed for the physical parameters they have considered, similar results are found in slightly different situations. Unfortunately, the enhanced trapping by the static magnetic field is only observed in a small range of the various values of the physical parameters. It seems that, close to the saturation level, when self-injection leads to the injection of a charge close to the maximal charge allowed by the beam-loading limit, adding a static magnetic field does not enhance the particle trapping.

It is shown that a current is created in a plane perpendicular to the direction of propagation of the wave which intensifies the initial static field which is applied.

Finally, it is confirmed in this paper that a constant magnetic field is a very important controlling knob for improving the charge of the beam in the LWFA process in the case of self-injection.

\section{REFERENCES}

[1] J. Faure, Y. Glinec, A. Pukhov, S. Kiselev, S. Gordienko, E. Lefebvre, J.-P. Rousseau, F. Burgy and V. Malka, "A Laser-Plasma Accelerator Producing Monoenergetic Electron Beams," Nature, Vol. 431, No. 7008, 2004, pp. 541544. doi:10.1038/nature02963

[2] E. Esarey, P. Sprangle, J. Krall and A. Ting, "Overview of Plasma-Based Accelerator," IEEE Transactions on Plasma Science, Vol. 24, 1996, pp. 252-288. doi:10.1109/27.509991

[3] Y. Glinec, J. Faure, L. Le Dain, S. Darbon, T. Hosokai, J. J. Santos, E. Lefevre, J. P. Rousseau, F. Burgy, B. Mercier and V. Malka, "High-Resolution-Ray Radiography Produced by a Laser-Plasma Driven Electron Source," Physical Review Letters, Vol. 94, No. 2, 2005, Article ID: 025003. doi:10.1103/PhysRevLett.94.025003

[4] G. A. Mourou, T. Tajima and S. V. Bulanov, "Optics in the Relativistic Regime," Reviews of Modern Physics, Vol. 78, No. 2, 2006, pp. 309-371. doi:10.1103/RevModPhys.78.309

[5] S. P. D. Mangles, C. D. Murphy, Z. Najmudin, A. G. R. Thomas, J. L. Collier, A. E. Dangor, E. J. Divall, P. S. Foster, J. G. Gallacher, C. J. Hooker, D. A. Jaroszynski, A. J. Langley, W. B. Mori, P. A. Norreys, F. S. Tsung, R. Viskup, B. R. Walton and K. Krushelnick, "Monoenergetic Beams of Relativistic Electrons from Intense Laser-Plasma Interactions," Nature, Vol. 431, No. 7008, 2004, pp. 535-538. doi:10.1038/nature02939

[6] C. G. R. Geddes, Cs. Toth, J. van Tilborg, E. Esarey, C. B. Schroeder, D. Bruhwiler, C. Nieter, J. Cary and W. P. Leemans, "High-Quality Electron Beams from a Laser Wakefield Accelerator Using Plasma-Channel Guiding," Nature, Vol. 431, No. 7008, 2004, pp. 538-541. doi:10.1038/nature 02900

[7] A. Zewail, "Atomic-Scale Dynamics of the Chemical Bond Using Ultrafast Lasers," Nobel Lecture 1996-2000, World Scientific Publishing Co., Singapore City, 2003, pp. 274-
367.

[8] T. Tajima and J. M. Dawson, "Laser Electron Accelerator," Physical Review Letters, Vol. 43, No. 4, 1979, pp. 267-270. doi:10.1103/PhysRevLett.43.267

[9] A. Pukhov and J. Meyer-ter-Vehn, "Laser Wake Field Acceleration: The Highly Non-Linear Broken-Wave Regime," Applied Physics B, Vol. 74, No. 4, 2002, pp. 355361. doi:10.1007/s003400200795

[10] W. Lu, C. Huang, M. Zhou, W. B. Mori and T. Katsouleas, "Nonlinear Theory for Relativistic Plasma Wakefields in the Blowout Regime," Physical Review Letters, Vol. 96, No. 16, 2006, Article ID: 165002.

doi:10.1103/PhysRevLett.96.165002

[11] J. Faure, C. Rechatin, A. Norlin, A. Lifschitz, Y. Glinec and V. Malka, "Controlled Injection and Acceleration of Electrons in Plasma Wakefields by Colliding Laser Pulses," Nature, Vol. 444, No. 7120, 2006, pp. 737-739. doi:10.1038/nature 05393

[12] A. I. Akhiezer and R. V. Polovin, "Theory of Wave Motion of an Electron Plasma," Soviet Physics, Vol. 3, No. 5, 1956, pp. 696-705.

[13] S. V. Bulanov, F. Pegoraro, A. M. Pukhov and A. S. Sakharov, "Transverse-Wake Wave Breaking," Physical Review Letters, Vol. 78, No. 22, 1997, pp. 4205-4208. doi:10.1103/PhysRevLett.78.4205

[14] M. S. Hur, D. N. Gupta and H. Suk, "Enhanced Electron Trapping by a Static Longitudinal Magnetic Field in Laser Wakefield Acceleration," Physics Letters A, Vol. 372 , No. 15,2008 , pp. 2684-2687. doi:10.1016/j.physleta.2007.12.045

[15] J. Vieira, S. F. Martins, V. B. Pathak, R. A. Fonseca, W. B. Mori and L. O. Silva, "Magnetic Control of Particle Injection in Plasma Based Accelerators," Physical Review Letters, Vol. 106, No. 22, 2011, Article ID: 225001. doi:10.1103/PhysRevLett.106.225001

[16] E. Lefebvre, N. Cochet, S. Fritzler, V. Malka, M.-M. Aléonard, J.-F. Chemin, S. Darbon, L. Disdier, J. Faure, A. Fedotoff, O. Landoas, G. Malka, V. Méot, P. Morel, M. Rabec Le Gloahec, A. Rouyer, Ch. Rubbelynck, V. Tikhonchuk, R. Wrobel. P. Audebert and C. Rousseaux, "Electron and Photon Production from Relativistic LaserPlasma Interactions," Nuclear Fusion, Vol. 43, No. 7, 2003, pp. 629-633. doi:10.1088/0029-5515/43/7/317

[17] J. P. Verboncoeur, A. B. Langdon and N. T. Gladd, "An Object-Oriented Electromagnetic PIC Code," Computers Physics Communications, Vol. 87, No. 1, 1995, pp. 199211. doi:10.1016/0010-4655(94)00173-Y

[18] A. Bourdier, S. Rassou, G. Girard and M. Drouin "Influence of a Magnetic Guide Field on Injection in Wakefield Acceleration," Journal of Modern Physics, Vol. 3, No. 9, 2012, pp. 1018-1020. doi:10.4236/jmp.2012.39133

[19] G. Gordienko and A. Pukhov, "Scalings for Ultrarelativistic Laser Plasmas and Quasimonoenergetic Electrons," Physics of Plasmas, Vol. 12, No. 4, 2005, Article ID: 043109. doi:10.1063/1.1884126

[20] W. Lu, M. Tzoufras, C. Joshi, F. S. Tsung, W. B. Mori, J. Vieira, R. A. Fonseca and L. O. Silva, "Generating Multi$\mathrm{GeV}$ Electron Bunches Using Single Stage Laser Wake- 
field Acceleration in a 3D Nonlinear Regime," Physical Review Special Topics-Accelerator and Beams, Vol. 10, No. 6, 2007, Article ID: 061301.

[21] S. Wilks, T. Katsouleas, J. M. Dawson and J. J. Su,
"Beam Loading Efficiency in Plasma Accelerators," Proceedings of the 1987 IEEE Particle Accelerator Conference: Accelerator Engineering and Technology, Vol. 1, 1987, pp. 100-102. 